\title{
Role of CRISPR-Cas/9 in Hematological Disorders: A Review
}

\author{
Seema Rani Padhiary ${ }^{1}$, Sameer Sharma ${ }^{1 *}$ \\ Department of BioNome, BioNome Private Limited, Bangalore, India
}

*Corresponding author: Sameer Sharma, Department of Bioinformatics, BioNome Private Limited, Bangalore, India.
Received Date: April 20, 2020

Published Date: May 20, 2021

\begin{abstract}
The ongoing research in genome editing and gene silencing methods have paved a number of ways in not just detecting the diseases but also in rectifying it too. CRISPR Cas9 technology has been a gift in the field of genome editing technology. CRISPR Cas9 not only helps in identifying the diseases located in a particular gene but also helps in correcting the gene in its genome level by either silencing the gene for generations or replacing the defective gene and modifying it. With the help of CRISPR Cas9 replacing concept the defective gene is replaced with the new one and the disorder is not continued further. This advancement in the technology is the key benefit for the CRISPR Cas9 procedure. To add with it zincfinger nucleases (ZFNs), transcription activator-like effector nucleases (TALENs)are the other two technology to contribute in genome editing too. Haematological disorders being lethal in nature have found a cure via CRISPR Cas9 technology.
\end{abstract}

Keywords: Clustered Regularly Interspaced Short Palindromic Repeats (CRISPR)-associated protein 9 (Cas9) system; Haematological disorders; Gene editing; Haematological malignancies \& monogenic

\section{Introduction}

Over the past two decades, advances in molecular biology and genetics have greatly extended our knowledge of hematological diseases. Every year hematological disease are affecting million people from malignancies to acquired coaglupathiesis. However, the characterization of genes of interest remains a great challenge. The optimal way to unravel the roles of particular genes is to manipulate their functions via gene deletion, insertion or modification. Genome engineering is defined as the deliberate modification of an organism's genetic material. From the 1980s several studies unravel the genetic mutation and their effect on human disease to determine the malignancies and advance diagnosis, prevention and diagnosis. Earlier gene editing technology were homologous recombination which was less efficient, time consuming \& laborintensive process. Later in subsequent years, Zinc finger nucleases [ZFNs] \& transcription activator-like effector nucleases [TALENs], was introduced to stimulate cellular DNA repair pathways. But there are so many limitations, so to overcome those limitation CRIPR associated CAS9 protein has introduce to start a new era of genome editing. From past few years CRISPR have change the path of genome editing be developing plant and animal models and the alleviation of human disease like hematological disorders. According to research crRNA sequence have significance role in targeting DNA [1,2]. To established CRISPR/Cas9 Jinek et al, 2012 introduce crRNA and tracrRNA into a single 100 nucleotide and a guider RNA which helps in the breaking of double stranded DNA and thus successfully genome editing done in human and mouse cells [3]. In the hematopoietic setting, CRISPR/Cas9 gene editing has been applied both in research and in clinical translation studies. CRISPER/Cas9 can be used to treat the clonal hematopoiesis by recapitulate the genetic mutation in patient. The main goal is to restore the immune system by the next generation genomics studies coupled with CRISPR/Cas9. Several companies develops their 
therapeutics against auto immune diseases and multiple myeloma by using this editing tools. The question marks comes whether this technique is safe or not, for this clinical trials are ongoing. The basic principle of CRISPR/Cas9 mediate gene modification are 1. Cas9 protein with the help of tracrRNA binds with specific DNA target which is marked by guider RNA with crRNA sequence. 2. Cas9 have endonuclease activity that helps in cleave of DNA. 3. The cleavage site will be repaired by the nonhomologous end joining (NHEJ) DNA repair pathway, by this process insertion/deletion can be done to make the gene functional or dysfunctional.

\section{CRISPR Cas/9: A Multifaceted tool for Genome ed- iting}

Clustered regularly interspersed short palindromic repeats (CRISPRs) were originally discovered in E. coli in 1987 [2,4,5]. The CRISPR-Cas systems are divided into two classes based on the structural variation of the Cas genes and their organization style[2] Specifically class 1 CRISPR-Cas systems consist of multiprotein effector complexes, whereas class 2 systems comprise only a single effector protein; altogether, six CRISPR-Cas types and at least 29 subtypes have been reported [6,7], and the list is rapidly expanding.
Type II CRISPR system is used in most of the cases which is depend on single CAS protein developd fron streptococcus targeting DNA and therefore active the gene editing tools [8] Mechanistically, the CRISPR/Cas9 system comprises two components, a singlestranded guide RNA [sgRNA] and a Cas9 endonuclease. To target the DNA need specific target sequence, thus the sgRNA consist of $20 \mathrm{BP}$ and this must be compatible with Cas9 protein used, which is termed the "protospacer adjacent motif" [PAM] of an "NGG" or "NAG" $[9,10]$. The sgRNA binds to the target sequence by WatsonCrick base pairing and Cas9 precisely cleaves the DNA to generate a DSB [1]. Following the DSB, DNA-DSB repair mechanisms initiate genome repair. Targeted genomic modification can be done with CRISPR/Cas9 through NHEJ or high fidelity HDR [11]. On the other way CISPR/Cas9 with guider RNA have more advantages then other editing system [12]. Endonuclease based ZFN or TALEN tools need to be reengineering for each case of targeting site, however the Cas 9 also be engineered to recognized new site via changing the guider RNA sequence. Another one advantages of CRISPR is that it has potential to multiple loci editing by changing the sequence which make its more suitable then other modification system (Figure 1).

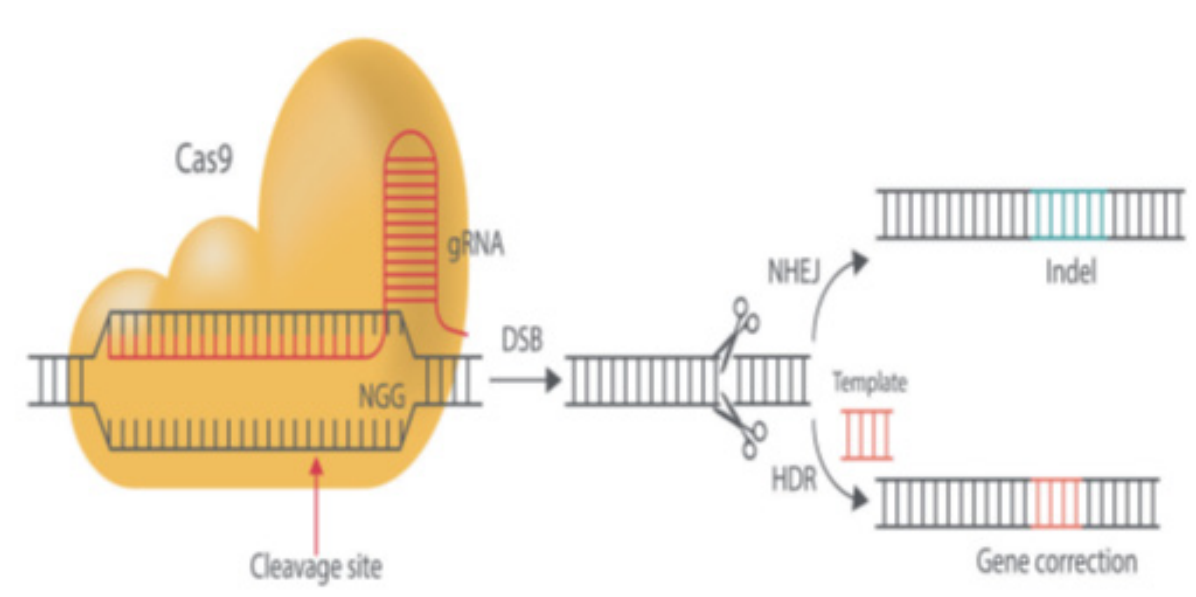

Figure 1: Illustration of CRISPR/Cas9 system.

CRISPR/Cas9 is now an indispensable tool in biological research. Three common strategies have been developed for genome editing with the CRISPR/Cas9 platform (1) The plasmid-based CRISPR/ Cas 9 strategy, where a plasmid is used to encode Cas9 protein and sgRNA, $[3,13]$ assembles Cas9 gene as well as sgRNA into the same plasmid in vitro. This strategy is longer lasting in the expression of Cas9 and sgRNA, and it prevents multiple transfections [14]. However, the plasmid needs to be introduce inside the nuclease of target cells.

The ethical and security risks comes under the consideration when gene editing done because it is irreversible. Some times its causes complicated nerves disease. As result genome editing can be done only for RNA modification by proposal of scientist. The byproduct of transcription, RNA is responsible for downstream processing of protein. RNA modification is one of the best methods to treat the disease to avoid the irreversible modification and mutation. In therapeutic genetics CRISPR can be introduce with the stem cell therapy. Pluripotent stem cells have ability to differentiate in retinal precursors, and it has been proven that CRIS with stem cells have less immune rejection problems [19.20]. However, patient-derived iPSCs might still harbor the same pathogenic genes, which could influence the therapeutic efficacy of transplanted cells.

ITherefore, it is necessary to combine the CRISPR/Cas9 system to fix disease- causing mutations in patient derived iPSCs before transplantation [21]. CRISPR was first described in Escherichia coli as an odd 29-nucleotide repeat sequence with 32-nucleotide spacing by a Japanese research group in 1987, although no further detailed observation was reported [22]. Several years later, Francisco Mojica 
discovered similar repeats in several microbial species and reported their potential use in prokaryotes [23]. By 2002, the novel repeats were formally named as Clustered Regularly Interspaced Short Palindromic Repeats [CRISPR] meanwhile, scientists discovered specific CRISPR-associated (Cas) genes that were closely related to the function of CRISPR [24]. In 2003, Mojica discovered the function of the CRISPR system, proposing that CRISPR is an adaptive immune system that protects microbes against specific infections [23]. Two independent groups $[4,5]$ reported similar conclusions, and their finding was confirmed by experiments showing that CRISPR provides acquired immunity against viruses in prokaryotes [25]. Researchers from the Massachusetts Institute of Technology have developed and optimized the Streptococcus pyogenes CRISPR-Cas9 system to facilitate efficient genome editing in mammalian cells [3]. Another group established a CRISPR-Cas9 system as an RNAguided editing tool for human genome engineering using a chimeric single-guide RNA [sgRNA] instead of a tracrRNA:crRNA duplex.

A CRISPR-Cas9 system was applied to screen for loss-offunction using a high-throughput screening platform in human cells

Table 1: Comparison of the most widely used Cas nucleases.
[26]. These genome-scale knockout screens provide an alternative screening system to RNA interference (RNAi), which is limited by partial depletion of target gene levels, toxicity and transient silencing [27]. [28] A catalytically inactive Cas9 protein [dCas9] was demonstrated lacking endonuclease activity that can be used as a platform for RNA-guided transcription regulation; this modified system is called CRISPR interference [Crispi]. Unlike RNAi-based silencing, which destructs transcribed mRNAs, the CRISPR-dCas9 system directly blocks transcription elongation within proteincoding regions and leads to dramatic suppression of transcription, with no detectable off-target effects [28]. The authors subsequently reported that both repressive and activating effector domains, such as KRAB and VP64, can be fused to dCas 9 to either repress [CRISPRi] or activate [CRISPRa] the transcription of target genes [29]. Other than targeting dsDNAs, CRISPR-Cas9 was modified to bind or cut RNA targets in vitro by providing the proto spacer adjacent motif [PAM] as part of an oligonucleotide [PAMmer]. RNA-targeting Cas9 [RCas9] as a tool to track RNAs in living cells, in which RCas9 is capable of tracking the localization and movement of endogenous RNAs (Table 1).

\begin{tabular}{|c|c|c|c|c|}
\hline Cas Nuclease & Identified from & Targeted Molecule & Key features & Most Frequent Application \\
\hline Cas9 & Streptococcus pyogenes & DNA & $\begin{array}{l}\text { DSB proximal to PAM (blunt ends). } \\
\text { Widely used in genome editing. } \\
\text { More off-targets than Cas } 9 \text { variants. }\end{array}$ & $\begin{array}{l}\text { Knock-out } \\
\text { Knock-in }\end{array}$ \\
\hline dCas9 & Mutant form of Cas 9 & DNA & $\begin{array}{c}\text { Lacks endonuclease activity. } \\
\text { Works by recruiting enhancers, silencers, } \\
\text { chromatin modifiers. Useful for single base } \\
\text { genome mutagenesis }\end{array}$ & $\begin{array}{l}\text { Regulation of gene expression } \\
\text { (CRISPRi/CRISPRa) }\end{array}$ \\
\hline Cas9 nickase & Mutant form of Cas 9 & DNA & $\begin{array}{c}\text { Single-strand break. } \\
\text { One inactive nuclease domain. } \\
\text { Higher accuracy in gene integration using two } \\
\text { nickases. } \\
\text { Lower off-targets tham Cas9. }\end{array}$ & Knock-in \\
\hline Cas12a (cpf1) & $\begin{array}{l}\text { Acidaminococcus sp. } \\
\text { Lachnospiraceae } \\
\text { bacterium }\end{array}$ & DNA & $\begin{array}{l}\text { DSB distal to PAM (staggered ends). } \\
\text { Cleaves first the non-target strand. } \\
\text { No requirement for tracrRNA. }\end{array}$ & $\begin{array}{l}\text { Knock-out } \\
\text { Knock-in }\end{array}$ \\
\hline Cas13a(C2c2) & $\begin{array}{l}\text { Leptotrichia wadei } \\
\text { Leptotrichia buccalis } \\
\text { Leptotrichia shahii }\end{array}$ & RNA & $\begin{array}{l}\text { Lacks a DNase domain. } \\
\text { No requirement for HDR machinery or a PAM. } \\
\text { Acts in non-dividing cells. } \\
\text { Cleaves additional RNA(only in bacteria). }\end{array}$ & Regulation of gene expression \\
\hline
\end{tabular}

For treating the hematopoietic cells, the main problem is efficiency of nontoxic delivery of components into therapeutic relevant cells like stem cells and $\mathrm{T}$ cells. However, the balancing need to be required the CAS9 RNP act in duration as short as possible. According to the several studies prolonged expression leads to higher chances of unintended activity at closely match target sequence. In general, there are different methods to deliver the CRISPR/Cas9 in the system.

- The 1st method DNA plasmid-based CRISPR/Cas9 system, which encodes the Cas9 protein and sgRNA from the same plasmid.

- The 2nd method 'all RNA'-based delivery method which Cas9-encoding mRNA and the sgRNA are delivered simultaneously.

- The last approach is delivery as an RNP complex consisting of recombinant Cas9 protein complexed to the sgRNA prior to delivery. Transfection with a plasmid-based CRISPR/Cas9 system has been extensively used in easy-to-manipulate cells. However, the major problem with this system is the induction of an innate immune response to foreign DNA, which is lacking in many cancer cell lines. A forementioned plasmid delivery in haematopoietic cells increases the cellular toxicities $[118,119]$. Electroporation with RNP complexes or Cas 9 mRNA and sgRNA have viewed to work very systematic in both the cells along with RNP system giving slightly higher INDEL frequencies [119,120,121]. Mordern transcriptomic profiling after Cas9 
mRNA transfer also suggests that mRNA does, to some extent, invoke an innate immune response in HSCs, which is not the case for Cas9 RNP delivery [122]. One limitation of this delivery modality is the apparent instability of the sgRNA, which leads to relatively low gene editing rates, particularly in primary cells [119].

\section{Applications of Crispr/Cas9 in Haematological Dis- orders}

The simplest genome editing system is type II CRISPR tool which require Cas9 protein and tracrRNA and crRNA [2,30]. This duplex is simplified by sgRNA and Cas 9 binds with double standard DNA and cleave it by endonuclease activity. Thus, it trigger NHEJ or HDR mediated genome editing [2]. The functions and mechanisms of the type II CRISPR-Cas9 system have been extensively reviewed in detail [31-33].

\section{Non-cancerous haematological disorders}

\section{Beta-thalassaemia}

b-thelassaemia caused by mutation in the human hemoglobin beta [HBB,also known as b-globin] and this is most common inherited blood disorders. The decreasing expression of HBB resulting erythropoiesis and thus severe anemia [34,35]. To treat this disorderd allogenicHSCT therapy is present previously but in resent era CRISPR Cas9 mediated gene editing is used to correct the HBB gene mutation via HDR. Apart from that stem cell also introduced to treat this erythropoiesis [37-39]. Gene-corrected iPSCs can restore HBB expression with a minimal off-target effect. In a recent study, the fibroblasts of a b-thalassaemia patient were reprogrammed to become transgene-free natıve-state iPSCs, which showed significantly higher targeting efficiencies in the CRISPRCas9 genome editing system compared with primed iPSCs $[40,41]$ Unfortunately, therapy via $\mathrm{HbF}$ reactivation has lagged for many years due to the unclear regulatory relationship between $\mathrm{HbF}$ and adult haemoglobin [HbA] during the development of erythroid cells. Recently [42] identified an erythroid-enhancer region within the BCL11A gene via CRISPR-Cas9-mediated in situ saturating mutagenesis. As an ideal therapeutic target for b-haemoglobin disorders, this region can be disrupted by individual sgRNAs, leading to efficient $\mathrm{HbF}$ reactivation.

\section{Sickle-cell disease}

SCD is another genetic disorder caused by deficiency of oxygen [43] it affects the red blood cells and form a sickle shape, clogs blood vessels and shows pain full symptoms like such as haemolysis, ischaemia, anaemia and multi-organ injury [44,45]. Several research attempts to correct the mutation by ZFN and TALEN induced HDR with the help of stem cells $[46,47]$. One recent study utilized a CRISPR-Cas9 system to target the endogenous HBB locus in human iPSCs generated from SCD patients, resulting in higher efficiencies compared with other nucleases [48]. In addition BCL11 gene targeting by CRISPR-Cas9 also can used to treat this [49].

\section{Thrombocytopenia}

Thrombocytopenia is an inherited or acquired alteration of genes, its lead to decreasing of thrombocytes [50]. Till now only few cases are documented as CRISPR treatment [51]. According to recent studies CRISPR-Cas9 gene editing to convert megakaryocytelike cells and iPSCs from HPA-1a (human platelet alloantigen-1a) to the HPA- $1 \mathrm{~b}$ alloantigenic epitope, it's a tough technique.

\section{Haemophilia}

Hemophelia is one of the common hereditary disorders of blood coagulation [52]. Hemophilia A caused by in the deficiency of factor viii and hemophilia B caused by deficiency of factor IX. There are several approaches done through Adenovirus-associated virus [AAV] vectors expressing to fix this $[53,54]$. Because AAV-mediated gene transfer is applicable only to a limited group of patients, alternative strategies have been proposed [High, 2012]. Last year, one research group developed a CRISPR-Cas9 system to revert two inverted chromosomal regions back to the normal orientation in HA patient-derived iPSCs.

\section{Malignant haematological disorders}

\section{Myeloma}

Worlds second most hematological disorder is multiple myeloma according to NHLs it happens because of malignant plasma cells in the bone marrow. According to research TALENs are used to treat MM [55], utilized CRISPR-Cas9 editing to silence MUC1-C, an oncogenic trans-membrane protein, and found that MUC1-C occupies the MYC promoter and activates the MYC gene via a b-catenin/TCF4-mediated mechanism. Further analysis using CRISPR-Cas9-mediated microarray datasets demonstrated that MUC1-C drives MYC expression and contributes to MM progression.

\section{Leukaemia}

In myelod lukaemia CRISPR editing has been overwhelmingly application [56]. Used the CRISPR-Cas9 system to generate mouse models of AML using lentivirus-mediated editing of multiple genes [e.g., Runx1 and Tp53, among others] in murine haematopoietic stem and progenitor cells [HSPC]. This work highlights the application of CRISPRCas 9 to generate ex vivo genome editing to delineate the complexity of human blood cancers. Later that year [57]. discovered a novel tumour suppressor gene, KMT2C [lysine methyltransferase 2C, also termed MLL3], in chromosome 7q using an ex vivo CRISPR-Cas9-mediated approach. Further experiments in a mouse model confirmed that KMT2C is a haplo insufficient tumour suppressor in AML and its inhibition impairs differentiation of the myeloid lineage and produces an MDS-like syndrome in mice. Interestingly, murine AMLs with KMT2C suppression are resistant to conventional chemotherapy. This study provides a valuable clue for the treatment of AML patients harbouring 7/del[7q] lesions. 


\section{Secondary haematological disorders}

\section{Human immunodeficiency virus (HIV) infection}

HIV infection has been associated with a broad range of haematological disorders [58]. ZFN-induced disruption of the CCR5 gene, an essential HIV-1 co-receptor required for virus entry, had become a promising gene therapy procedure [59]. CRISPR-Cas 9 have further transformed the studies of HIV-1 [60]. DNA division activity of a CRISPR-Cas9 system for silencing the CCR5 gene in vitro, have reported CRISPRCas9- mediated disruption of CCR5 [61,62] and other co-factors, including CXCR4 [63] and XPO1 exportin 1 [64]. The removable of pro-viral HIV-1 DNA from the host cell genome, have reported the potential of the CRISPR-Cas9 system to mutations by targeting the long terminal repeat [LTR] region [6568]. Moreover, the feasibility of HIV therapy has been questioned by the latest finding that viewed viral escape from CRISPR geneediting attack due to the Cas9/ sgRNA-derived [69]. CRISPR-dCas9derived regeneration of HIV-1 is specific in all latency models and does not induce off-target effects [70] (Figure 2).

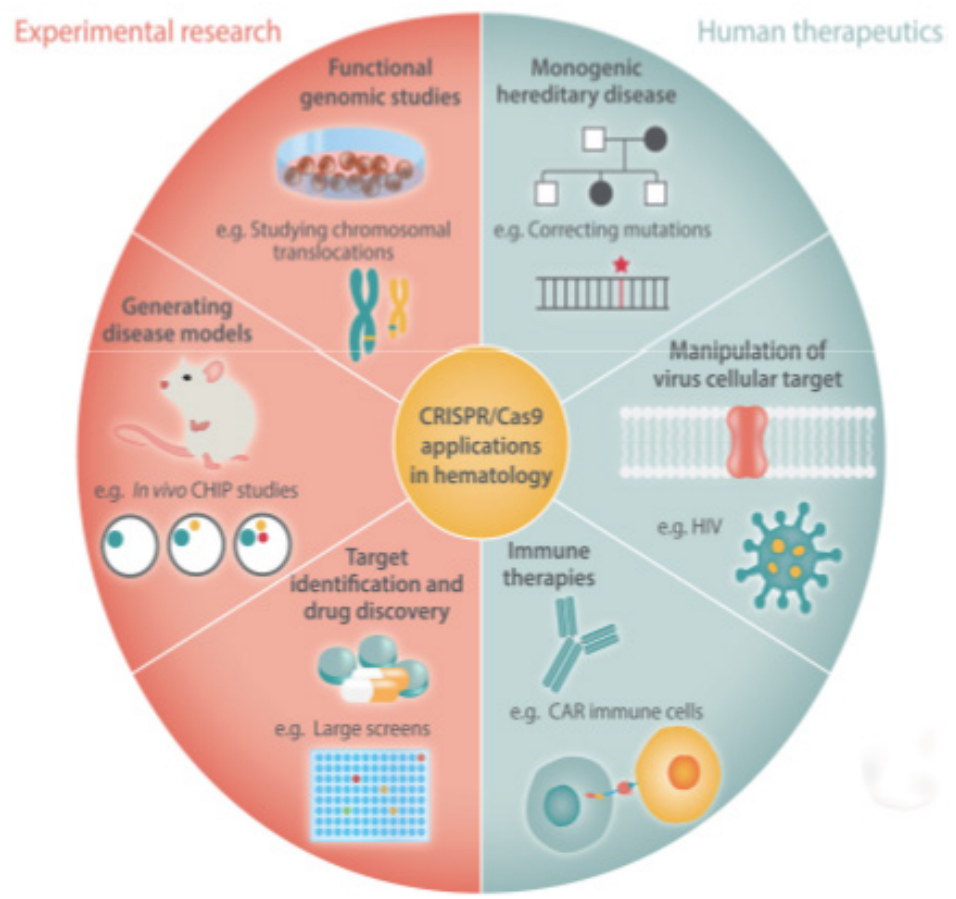

Figure 2: Applications of CRISPR/Cas9 in Hematology

\section{Applications of CRISPR/Cas9 in Hematology}

\section{Malaria}

Since the CRISPR-Cas9 was successfully applied in Drosophila in vivo [71]. scientists have attempted to apply to target either malaria parasites [72,73] or mosquitoes [71,72]. In a representative study, it was identified that the gene required for fertility in female Anopheles mosquitoes can be inserted via CRISPR-Cas9 gene-drive construct and targeting the loci [74]. This procedure made a kind of sterility in female mosquitoes, paving a way to extinction of the population. Parasite transmission was baffled by incorporating into an anti-malarial gene into malaria mosquitoes via the help of CRISPR-Cas9 [71]. This strategy however declined the graph of malaria but have not lead the diseases to extinct $[75,76]$.

\section{Technical restrictions}

CRISPR-Cas9-mediated gene enhancement can be gained by transient or stable transfer of the CRISPR components $[3,13,60]$,
Viral-based transfection of CRISPR components is a more efficient substitute to create stably modified cells $[56,77,78,79]$. [80] Development of rapid cell mechanical deformation has been developed which creates transient holes in the membrane that helps in the transfer of biomaterials through the medium. The MCL1, an anti-apoptotic BCL2 family member, is required for the sustained survival of human BL cell lines because silencing of MCL1 leads to rapid cell death. In this case, the drug-inducible CRISPRCas9 lentiviral system provides an option to identify the functions of tumour-essential genes [81]. Due to the increased utilization of the NHEJ pathway over HDR in mammalian cells, the low efficiency of CRISPRCas9- induced HDR is an additional limitation [13,82]. Therefore, increasing the efficiency of HDR facilitates the rapid generation of cell lines with precisely edited genes. To resolve this issue, a recent study identified a RAD51-stimulatory compound, RS-1, which enhances the efficiency of Cas9-stimulated HDR by 3to 6-fold [83]. 


\section{Off-target effects}

CRISPR-Cas9 in mammalian cells, have promising results but the question of specificity is still of major concern [84-88]. Deep sequencing $[85,86]$ and whole genome sequencing $[89,90]$ have been considered options to identify mismatches however, the possibility of overlooking off-target sites and the higher cost make it difficult to perform this step in every experiment. Alternatively, a series of online bioinformatics tools were developed to predict off-target mutation sites [11,92-94]. Genome-wide Unbiased recognition of DSBs authorize by Sequencing (GUIDE-seq) provides an unbiased and genome-wide method for identifying CRISPR RNA-guided DSBs in cells $[91,95,96]$. These studies have enlarged the technical feasibility of off-target detection $[97,98]$, although 'it is impossible to draw broad conclusions about the specificity of engineered nucleases', as recently reported [99,100] (Table 2).

Table 2: Current clinical trials in hematology using the CRISPR/Cas9 system.

\begin{tabular}{|c|c|c|c|c|c|}
\hline Disease & Product & Aim/Title & Phase & CT identifier & Industry/Academy \\
\hline$\beta$ thalassemia & $\begin{array}{l}\text { CTX001 } \\
\text { Ihsc treatment } \\
\text { group }\end{array}$ & $\begin{array}{l}\text { A safety \& efficacy study evaluating } \\
\text { CTX001 in subjects with transfusion- } \\
\text { dependent } \beta \text { thalassemia. } \\
\text { iHSC with the gene correction of HBB } \\
\text { intervent subjects with } \beta \text { thalassemia } \\
\text { mutations. }\end{array}$ & $\begin{array}{l}\text { Enrolling } \\
\text { Not yet } \\
\text { recruting }\end{array}$ & $\begin{array}{l}\text { NCT03655678 } \\
\text { 2017-003351-38 } \\
\text { NCT03728322 }\end{array}$ & $\begin{array}{l}\text { CRISPR Therapeutics. } \\
\text { Allife Medical Science and } \\
\text { Technology. }\end{array}$ \\
\hline $\begin{array}{c}\text { CD19+ leukemia CD19 } \\
\text { + lymphoma }\end{array}$ & СTX110 & $\begin{array}{l}\text { Anti-CD19 allogeneic CAR-T cells } \\
\text { with TCR and B2M knocked-out. }\end{array}$ & $\begin{array}{l}\text { Initiates in } \\
\text { first half of } \\
2019\end{array}$ & NA & CRISPR Therapeutics. \\
\hline HIV-1 infection & $\begin{array}{l}\text { CCR5 gene } \\
\text { modification }\end{array}$ & $\begin{array}{l}\text { Safety of transplantation of CRISPR } \\
\text { CCR5 modified CD34+ HSPC in HIV- } \\
\text { infected subjects with hematologic } \\
\text { malignancies. }\end{array}$ & Enrolling & NCT03164135 & Beijing, China. \\
\hline $\begin{array}{l}\text { B-cell leukemia } \\
\text { B-cell lymphoma }\end{array}$ & $\begin{array}{l}\text { UCART019 } \\
\text { CTX101 }\end{array}$ & $\begin{array}{l}\text { A study evaluating UCART019 in } \\
\text { patients with relapsed or refractory } \\
\text { CD19+ leukemia \& lymphoma. } \\
\text { A feasibility\& safety study } \\
\text { of universal dual specificity } \\
\text { CD19 \&CD20 or CD2 CAR-T cell } \\
\text { immunotherapy. }\end{array}$ & $\begin{array}{l}\text { Phase I/II } \\
\text { Phase I/II }\end{array}$ & $\begin{array}{l}\text { NCT03166878 } \\
\text { NCT03398967 }\end{array}$ & $\begin{array}{l}\text { Beijing, China. } \\
\text { Beijing, China. }\end{array}$ \\
\hline Multiple myeloma & $\begin{array}{l}\text { NYCE T Cells } \\
\text { CTX120 }\end{array}$ & $\begin{array}{l}\text { NY-ESO-1-redirected CRISPR edited } \\
\text { T cells. } \\
\text { Anti-BCMA allogeneic CAR-T-cells } \\
\text { with TCR\& B2M knocked-out. }\end{array}$ & $\begin{array}{l}\text { Preclinical } \\
\text { Research }\end{array}$ & $\begin{array}{c}\text { NCT03399448 } \\
\text { NA }\end{array}$ & $\begin{array}{l}\text { Pennsylvania, USA. } \\
\text { CRISPR Therapeutics. }\end{array}$ \\
\hline $\begin{array}{c}\text { T-cell ALL } \\
\text { T-cell lymphoblastic } \\
\text { lymphoma } \\
\text { T-non-Hodgkin } \\
\text { lymphoma }\end{array}$ & $\begin{array}{l}\text { CD7.CAR/28zeta } \\
\text { CAR-T cells }\end{array}$ & $\begin{array}{l}\text { Cell therapy for high risk T-cell } \\
\text { malignancies using CD7-specific } \\
\text { CAR expressed on autologous. T } \\
\text { cells(CRIMSON) }\end{array}$ & Phase I & NCT03690011 & Houston, USA. \\
\hline
\end{tabular}

\section{Consequences and Future Perspectives}

CRISPR-technologies have been flourished beyond genome editing while simultaneously research is going on, either NHEJ or HR using then conventional CRISPR/Cas9 platform. The superiority of the procedure is that it helps in converting of one base pair with another without instigating any DSBs and without depending on the HR pathway or systematic delivery of a DNA donor template. The vivo HSC gene therapy with retroviral vectors has given a promising result with [101-103], CRISPR/Cas9 gene editing in a mouse model of b-thalassaemia also [104]. The pre-existing immunity in homo sapiens is potentially an obstacle Cas9 in humans $[105,106]$, which may be avoided by administration of immunosuppressive drugs [107]. CRISPR Cas9 has shown positive results in the field of genome editing and complete gene silencing technology. The utility of this technology in the field of haematological disorders have saved a number of lives. CRISPR Cas9 is a modern technique which uses bioinformatic tools to locate the mutated gene, and to top it even helps in rectifying it too. The use of this technology in curing haematopeotic cells are very promising and have a wider prospective in future too, to deal with any clinical manifestations.

\section{Acknowledgement}

None.

\section{Conflicts of Interest}

No conflicts of interest.

\section{References}

1. Gasiunas, G Barrangou, R Horvath, P Siksnys (2012) Cas9-crRNA ribonucleoprotein complex mediates specific DNA cleavage for adaptive immunity in bacteria. Proceedings of the National Academy of Sciences of the United States of America 109: E2579-E2586.

2. Jinek M, Chylinski, K Fonfara, I Hauer, M Doudna, et al. (2012) A programmable DNA endonuclease in adaptive bacterial immunity. Science 337: 816-821. 
3. Cong L, Ran FA Cox, D Lin, S Barretto (2013) Multiplex genome engineering using RISPR/Cas systems. Science 3396(121): 819-823.

4. Bolotin, Inquis B, Sorokin A, Ehrlich SD (2005) Clustered regularly interspaced short palindrome repeats CRISPRs have spacers of extrachromosomal origin. Microbiology Reading 151(8): 2551-2561.

5. Pourcel C, Salvignol G, Vergnaud (2005) CRISPR elements in Yersinia pestis acquire new repeats by preferential uptake of bacteriophage DNA and provide additional tools for evolutionary studies. Microbiology Reading 151(3): 653-663.

6. Makarova KS, Yuri I Wolf, Omer S Alkhnbashi, Fabrizio Costa, Shiraz A Shah, et al. (2015) An updated evolutionary classification of CRISPR-Cas systems. Nat Rev Microbiol 13(11): 722-736.

7. Makarova KS, Daniel H Haft, Rodolphe Barrangou, Stan J J Brouns, Emmanuelle Charpentier, et al. (2011) Evolution and classification of the CRISPR-Cas systems. Nat Rev Microbiol 9(6): 467-477.

8. Jiang W, Bikard D, Cox D, Zhang F, Marraffini LA (2013) RNA-guided editing of bacterial genomes using CRISPR-Cas systems. Nat Biotechnol 31: 233-239.

9. Sternberg SH, Redding S, Jinek M, Greene EC, Doudna JA (2014) DNA interrogation by the CRISPR RNA-guided endonuclease Cas9. Nature 507: $62-67$

10. Deveau H, Rodolphe Barrangou, Josiane E Garneau, Jessica Labonte, Christophe Fremaux, et al. (2008) Phage response to CRISPR-encoded resistance in Streptococcus thermophilus. J Bacteriol 190(4): 1390 1400.

11. Ran FA, Patrick D Hsu, Jason Wright, Vineeta Agarwala, David A Scott, et al. (2013) Genome engineering using the CRISPR-Cas9 system. Nat Protoc 8(11): 2281-2308.

12. Cox D, B Platt RJ, Zhang F (2015) Therapeutic genome editing prospects and challenges. Nat Med 21(2): 121-131.

13. Mali P, Luhan Yang, Kevin M Esvelt, John Aach, Marc Guell, et al. (2013) RNA-guided human genome engineering via Cas9. Science 339(6121): 823-826.

14. Doudna JA, Charpentier E (2014) Genome editing. The new frontier of genome engineering with CRISPR-Cas9. Science 346(6213): 1258096

15. Liu C, Zhang L, Liu H, Cheng K (2017) Delivery strategies of the CRISPRCas9 gene editing system for therapeutic applications. J Control Release 266: 17-26.

16. Biagioni A (2018) Delivery systems of CRISPR/Cas9-based cancer gene therapy. J Biol Eng 12: 33.

17. Abudayyeh 00, Jonathan S Gootenberg, Patrick Essletzbichler, Shuo Han, Julia Joung, et al. (2017) RNA targeting with CRISPR-Cas13. Nature 550(7675): 280-284.

18. Merkle T, Sarah Merz , Philipp Reautschnig, Andreas Blaha, Qin Li, et al. (2019) Precise RNA editing by recruiting endogenous ADARs with antisense oligonucleotides. Nat Biotechnol 37(2): 133-138.

19. Tucker BA, In-Hyun Park, Sara D Qi, Henry J Klassen, Caihui Jiang, et al. (2011) Transplantation of adult mouse iPS cell-derived photoreceptor precursors restores retinal structure and function in degenerative mice. PloS ONE 6(4): e18992.

20. Homma K, Satoshi Okamoto, Michiko Mandai, Norimoto Gotoh, Harsha K Rajasimha, et al. (2013) Developing rods transplanted into the degenerating retina of Crx-knockout mice exhibit neural activity similar to native photoreceptors. Stem Cells Dayton OH 31(6): 1149-1159.

21. Cai, B, Shuo Sun, Zhiqing Li, Xiaomin Zhang, Yifeng Ke, et al. (2018) Application of CRISPR/Cas9 technologies combined with iPSCs in the study and treatment of retinal degenerative diseases. Hum Genet 137(9): 679-688

22. Ishino Y, Shinagawa H, Makino K, Amemura M, Nakata A(1987) Nucleotide sequence of the iap gene, responsible for alkaline phosphatase isozyme conversion in Escherichia coli, and identification of the gene product. Journal of Bacteriology 169(12): 5429-5433.
23. Mojica FJ, Juez G, Rodriguez-Valera F (1993) Transcription at different salinities of Haloferax mediterranei sequences adjacent to partially modified PstI sites. Molecular Microbiology 9(3): 613-621.

24. Jansen R, Embden JD, Gaastra W, Schouls LM (2002) Identification of genes that are associated with DNA repeats in prokaryotes. Molecular Microbiology 43(6): 1565-1575.

25. Barrangou R, Fremaux C, Deveau H, Richards M, Boyaval P, et al. (2007) CRISPR provides acquired resistance against viruses in prokaryotes. Science 315(5819): 1709-1712.

26. Koike-Yusa H, Li Y, Tan EP, Velasco-Herrera M del C, Yusa (2014) Genomewide recessive genetic screening in mammalian cells with a lentiviral CRISPR-guide RNA library. Nature Biotechnology 32(3): 267-273.

27. Echeverri CJ, Beachy PA, Baum B, Boutros M, Buchholz, et al. (2006) Minimizing the risk of reporting false positives in large-scale RNAi screens. Nature Methods 3(10): 777-779.

28. Qi LS, Larson MH, Gilbert LA, Doudna JA, Weissman JS, et al. (2013) Repurposing CRISPR as an RNA-guided platform for sequence-specific control of gene expression. Cell 152(5): 1173-1183.

29. Gilbert LA, Larson MH, Morsut L, Liu Z, Brar (2013) CRISPR-mediated modular RNA-guided regulation of transcription in eukaryotes. Cell 154(2): 442-451.

30. Wiedenheft B, Lander GC, Zhou K, Jore MM, Brouns SJ van der Oost, et al. (2011) Structures of the RNA-guided surveillance complex from a bacterial immune system. Nature 477(7365): 486-489.

31. Hsu PD, Lander ES, Zhang F (2014) Development and applications of CRISPR-Cas9 for genome engineering. Cell 157(6): 1262-1278.

32. Sanchez-Rivera FJ, Jacks T (2015) Applications of the CRISPR-Cas9 system in cancer biology. Nature Review Cancer 15(7): 387-395.

33. Lander ES (2016) The heroes of CRISPR. Cell 164(1-2): 18-28.

34. Ribeil JA, Arlet JB, Dussiot M, Moura IC, Courtois, et al. (2013) Ineffective erythropoiesis in beta -thalassemia. Scientific World Journal 2013: 394 295.

35. Finotti A, Breda L, Lederer CW, Bianchi N, Zuccato C, et al. (2015) Recent trends in the gene therapy of beta-thalassemia. Journal of Blood Medicine 6: 69-85.

36. King A, Shenoy S (2014) Evidence-based focused review of the status of hematopoietic stem cell transplantation as treatment of sickle cell disease and thalassemia. Blood 123(20): 3089-3094.

37. Xie F, Ye L, Chang JC, Beye, AI, Wang et al. (2014) Seamless gene correction of beta-thalassemia mutations in patient-specific iPSCs using CRISPR/Cas9 and piggy Bac. Genome Research 24(9): 1526-1533.

38. Song B, Fan Y, He W, Zhu D, Niu, et al. (2015) Improved hematopoietic differentiation efficiency of gene corrected beta-thalassemia induced pluripotent stem cells by CRISPR/Cas9 system. Stem Cells and Development 24(9): 1053-1065.

39. Xu P, Tong Y, Liu XZ, Wang TT, Cheng, et al. (2015) Both TALENs and CRISPR/Cas9 directly target the HBB IVS2-654 C > T mutation in betathalassemia-derived iPSCs. Scientific Reports 5: 12065.

40. Yang Y, Zhang X, Yi L, Hou Z, Chen J, etal. (2016) Naive induced pluripotent stem cells generated from beta-thalassemia fibroblasts allow efficient gene correction with CRISPR/Cas9. Stem Cells Translational Medicine 5(1): 8-19.

41. Bauer DE, Kamran SC, Orkin SH (2012) Reawakening fetal hemoglobin: prospects for new therapies for the beta-globin disorders. Blood 120(15): 2945-2953.

42. Canver MC, Smith EC, Sher F, Pinello L, Sanjana NE, et al. (2015) BCL11A enhancer dissection by Cas9-mediated in situ saturating mutagenesis. Nature 527(7577): 192-197.

43. Weatherall DJ (2010) The inherited diseases of hemoglobin are an emerging global health burden. Blood 115(22): 4331-4336. 
44. Gewin V (2015) Medicine: expanding possibilities. Nature 528: S10-S11.

45. Hoban MD, Orkin SH, Bauer DE (2016) Genetic treatment of a molecular disorder gene therapy approaches to sickle cell disease. Blood 127(7): 839-848.

46. Zou J, Mali P, Huang X, Dowey SN, Cheng L (2011) Site-specific gene correction of a point mutation in human iPS cells derived from an adult patient with sickle cell disease. Blood 118(7): 4599-4608.

47. Sun N, Zhao H (2014) Seamless correction of the sickle cell disease mutation of the HBB gene in human induced pluripotent stem cells using TALENs. Biotechnology and Bioengineering 111(5): 1048-1053.

48. Huang X, Wang Y, Yan W, Smith C, Ye Z, et al. (2015) Production of genecorrected adult beta globin protein in human erythrocytes differentiated from patient iPSCs after genome editing of the sickle point mutation. Stem Cells, 33(5) 1470-1479.

49. Renneville A, Van Galen P, Canver MC, McConkey M, Krill-Burger JM, et al. (2015) EHMT1 and EHMT2 inhibition induces fetal hemoglobin expression. Blood 126(16): 1930-1939.

50. Drachman JG (2004) Inherited thrombocytopenia when a low platelet count does not mean ITP. Blood 103(2): 390-398.

51. Zhang N, Zhi H, Curtis B, Rao S, Jobaliya C, et al. (2016) CRISPR/Cas9mediated conversion of human platelet alloantigen allotypes. Blood 127(6): 675-680

52. Ratnoff OD, Bennett B, (1973) The genetics of hereditary disorders of blood coagulation. Science 179(4080): 1291-1298.

53. McIntosh J, Lenting PJ, Rosales C, Lee D, Rabbanian S, et al. (2013) Therapeutic levels of FVIII following a single peripheral vein administration of rAAV vector encoding a novel human factor VIII variant. Blood 121(7): 3335-3344

54. Nathwani AC, Tuddenham EG, Rangarajan S, Rosales C, McIntosh, et al (2011) Adenovirus-associated virus vector mediated gene transfer in hemophilia B. New England Journal of Medicine 365: 2357-2365.

55. Tagde A, Rajabi H, Bouillez A, Alam M, Gali, et al. (2016) MUC1-C drives MYC in multiple myeloma. Blood 127(21): 2587-2597.

56. Heckl D, Kowalczyk MS, Yudovich D, Belizaire R, Puram RV, et al. (2014) Generation of mouse models of myeloid malignancy with combinatorial genetic lesions using CRISPR-Cas9 genome editing. Nature Biotechnology32(9): 941-946.

57. Chen C, Liu Y, Rappaport AR, Kitzing T, Schultz N, et al. (2014) MLL3 is a haploinsufficient $7 q$ tumor suppressor in acute myeloid leukemia. Cancer Cell 25(5): 652-665.

58. Scadden DT, Zon LI, Groopman JE, (1989) Pathophysiology and management of HIV-associated hematologic disorders. Blood 7495): 1455-1463.

59. Tebas P, Stein D, Tang WW, Frank I, Wang SQ, et al. (2014) Gene editing of CCR5 in autologous CD4 T cells of persons infected with HIV. New England Journal of Medicine 370(10): 901-910.

60. Cho SW, Kim S, Kim JM, Kim JS (2013) Targeted genome engineering in human cells with the Cas9 RNA-guided endonuclease. Nature Biotechnology 31(3): 230-232.

61. Cradick TJ, Fine EJ, Antico CJ, Bao G (2013) CRISPR/Cas9 systems targeting beta-globin and CCR5 genes have substantial off-target activity. Nucleic Acids Research 41(20): 9584-9592.

62. Ye L, Wang J, Beyer AI, Teque F, Cradick TJ, et al. (2014) Seamless modification of wild-type induced pluripotent stem cells to the natura CCR5Delta32 mutation confers resistance to HIV infection. Proceedings of the National Academy of Sciences of the United States of America 111(26): 9591-9596

63. Hou P, Chen S, Wang S, Yu X, Chen Y, et al. (2015) Genome editing of CXCR4 by CRISPR/cas9 confers cells resistant to HIV-1 infection. Scientific Reports 5: 15577.
64. Boons E, Vanstreels E, Jacquemyn M, Nogueira TC, Neggers, et al. (2015) Human Exportin-1 is a target for combined therapy of HIV and AIDS related lymphoma. E Biomedicine 2(9): 1102-1113.

65. Ebina H, Misawa N, Kanemura Y, Koyanagi Y (2013) Harnessing the CRISPR/Cas9 system to disrupt latent HIV-1 provirus. Scientific Reports 3: 2510 .

66. Hu W, Kaminski R, Yang F, Zhang Y, Cosentino L, et al. (2014) RNAdirected gene editing specifically eradicates latent and prevents new HIV-1 infection. Proceedings of the National Academy of Sciences of the United States of America 111(31): 11461-11466.

67. Zhu W, Lei R, Le Duff Y, Li J, Guo F, (2015) The CRISPR/ Cas9 system inactivates latent HIV-1 proviral DNA. Retrovirology 12: 22.

68. Kaminski R, Chen Y, Fischer T, Tedaldi E, Napoli A, et al. (2016) Elimination of HIV-1 Genomes from Human T-lymphoid Cells by CRISPR/Cas9 Gene Editing. Scientific Reports 6: 22555.

69. Wang Z, Pan Q, Gendron P, Zhu W, Guo F, et al. (2016) CRISPR/Cas9derived mutations both inhibit HIV-1 replication and accelerate viral escape. Cell Report 15(3): 481-489.

70. Saayman SM, Lazar DC, Scott TA, Hart JR, Takahashi, et al. (2016) Potent and targeted activation of Latent HIV-1 using the CRISPR/dCas9 activator complex. Molecular Therapy 24(3): 488-498.

71. Gantz VM, Jasinskiene N, Tatarenkova O, Fazekas A, Macias V, et al. (2015) Highly efficient Cas9-mediated gene drive for population modification of the malaria vector mosquito Anopheles stephensi. Proceedings of the National Academy of Sciences of the United States of America 112: E6736-E6743.

72. Ghorbal M, Gorman M, Macpherson CR, Martins RM, Scherf et al. (2014) Genome editing in the human malaria parasite Plasmodium falciparum using the CRISPR-Cas9 system. Nature Biotechnology 32(8): 819-821.

73. Zhang C, Xiao B, Jiang Y, Zhao Y, Li (2014) Efficient editing of malaria parasite genome using the CRISPR/ Cas9 system. MBio 5(4): e0141401414.

74. Hammond A, Galizi R, Kyrou K, Simoni A, Siniscalchi, et al. (2016) A CRISPR Cas9 gene drive system targeting female reproduction in the malaria mosquito vector Anopheles gambiae. Nature Biotechnology 34(1) : 78-83.

75. Pennisi E (2015) SCIENCE AND SOCIETY. Gene drive turns mosquitoes into malaria fighters. Science 350(6264): 1014

76. Alphey L (2016) Can CRISPR-Cas9 gene drives curb malaria? Nature Biotechnology 34(2):149-150.

77. Malina A, Mills JR, Cencic R, Yan Y, Fraser, et al. (2013) Repurposing CRISPR/Cas9 for in situ functional assays. Genes \& Development 27(23): 2602-2614.

78. Chen Z, Teo AE, McCarty N (2016) ROSInduced CXCR4 Signaling Regulates Mantle Cell Lymphoma MCL cell survival and drug resistance in the bone marrow microenvironment via autophagy. Clinical Cancer Research 22(1): 187-199.

79. Zhang H, Chen Z, Neelapu SS, Romaguera J, McCarty N (2016) Hedgehog inhibitors selectively target cell migration and adhesion of mantle cell lymphoma in bone marrow microenvironment. Oncotarget 7(12): 14350-14365.

80. Han X, Liu Z, Jo MC, Zhang K, Li, Y, et al. (2015) CRISPR-Cas9 delivery to hard-to-transfect cells via membrane deformation. Science Advances 1(7): e1500454.

81. Aubrey BJ, Kelly GL, Kueh AJ, Brennan MS, O Connor (2015) An inducible lentiviral guide RNA platform enables the identification of tumoressential genes and tumor-promoting mutations in vivo. Cell Reports 10(8): 1422-1432.

82. Wang H, Yang H, Shivalila CS, Dawlaty MM, Cheng, et al. (2013) One-step generation of mice carrying mutations in multiple genes by CRISPR/ Casmediated genome engineering. Cell 153(4): 910-918. 
83. Pinder J, Salsman J, Dellaire G (2015) Nuclear domain 'knock-in' screen for the evaluation and identification of small molecule enhancers of CRISPR-based genome editing. Nucleic Acids Research 43(19): 93799392.

84. Fu Y, Foden JA, Khayter C, Maeder ML, Reyon, et al. (2013) Highfrequency off-target mutagenesis induced by CRISPR-Cas nucleases in human cells. Nature Biotechnology 31(9): 822-826.

85. Hsu PD, Scott DA, Weinstein JA, Ran FA, Konermann, et al. (2013) DNA targeting specificity of RNA-guided Cas9 nucleases. Nature Biotechnology 31: 827-832.

86. Mali P, Aach J, Stranges PB, Esvelt KM, Moosburner et al. (2013) CAS9 transcriptional activators for target specificity screening and paired nickases for cooperative genome engineering. Nature Biotechnology 31(9): 833-838.

87. Pattanayak V, Lin S, Guilinger JP, Ma E, Doudna JA, et al. (2013) High-throughput profiling of off-target DNA cleavage reveals RNAprogrammed Cas9 nuclease specificity. Nature Biotechnology 31: 839843.

88. Smith C, Gore A, Yan W, Abalde-Atristain L, Li Z, et al. (2014) Wholegenome sequencing analysis reveals high specificity of CRISPR/Cas9 and TALEN-based genome editing in human iPSCs. Cell Stem Cell 15(1): 12-13.

89. Veres A, Gosis BS, Ding Q, Collins R, Ragavendran A, et al. (2014) Low incidence of off-target mutations in individual CRISPR-Cas9 and TALEN targeted human stem cell clones detected by whole-genome sequencing. Cell Stem Cell 15(1): 27-30.

90. Wang X, Wang Y, Wu X, Wang J, Wang (2015) Unbiased detection of offtarget cleavage by CRISPR-Cas 9 and TALENs using integrase-defective lentiviral vectors. Nature Biotechnology 33(2): 175-178.

91. Fine EJ, Cradick TJ, Zhao CL, Lin Y, Bao G (2014) An online bioinformatics tool predicts zinc finger and TALE nuclease off-target cleavage. Nucleic Acids Research 42(6): e42.

92. Heigwer F, Kerr G, Boutros M (2014) ECRISP fast CRISPR target site identification. Nature Methods 11(20): 122-123.

93. Xie S, Shen B, Zhang C, Huang X, Zhang Y (2014) sgRNAcas9 a software package for designing CRISPR sgRNA and evaluating potential off-target cleavage sites. PLoS ONE 9(6): e100448.

94. Tsai SQ, Zheng Z, Nguyen NT, Liebers M, Topkar VV, (2015) GUIDE-seq enables genome wide profiling of off-target cleavage by CRISPR-Cas nucleases. Nature Biotechnology 33(2): 187-197.
95. Frock RL, Hu J, Meyers RM, Ho YJ, Kii E, et al. (2015) Genome-wide detection of DNA double-stranded breaks induced by engineered nucleases. Nature Biotechnology 33(2): 179-186.

96. Koo T, Lee J, Kim JS (2015) Measuring and reducing off-target activities of programmable nucleases including CRISPR-Cas9. Molecules and Cells 38(6): 475-481.

97. Bolukbasi MF, Gupta A, Wolfe SA (2016) Creating and evaluating accurate CRISPR-Cas9 scalpels for genomic surgery. Nature Methods 13(1): 41-50.

98. Gabriel R, Von Kalle C, Schmidt M (2015) Mapping the precision of genome editing. Nature Biotechnology 33(2): 150-152.

99. Ul Ain Q, Chung JY, Kim YH (2015) Current and future delivery systems for engineered nucleases ZFN, TALEN and RGEN. Journal of Controlled Release 205: 120-127.

100. Wang X, Shin SC, Chiang AF, Khan I, Pan D, et al (2015) Intraosseous delivery of lentiviral vectors targeting factor VIII expression in platelets corrects murine hemophilia A. Molecular Therapy 23(4): 617-626.

101. Wang H, Georgakopoulou A, Psatha N, Li C, Capsali, et al. (2018) In vivo hematopoietic stem cell gene therapy ameliorates murine thalassemia intermedia. Journal of Clinical Investigation 129(2): 598-615.

102. Richter M, Saydaminova K, Yumul R, Krishnan R, Liu J, et al. (2016) In vivo transduction of primitive mobilized hematopoietic stem cells after intravenous injection of integrating adenovirus vectors. Blood 128(18): 2206-2217.

103. Li C, Psatha N, Sova P, Gil S, Wang H, et al. (2018) Reactivation of gammaglobin in adult beta- YAC mice after ex vivo and in vivo hematopoietic stem cell genome editing. Blood 131(26): 2915-2928.

104. Charlesworth CT Deshpande PS, Dever DP, Dejene B, Gomez-Ospina, et al. (2018) Identification of preexisting adaptive immunity to Cas9 proteins in humans. Bio Rxiv 25: 243345.

105. Wagner DL, Amini L, Wendering DJ, Burkhardt LM, Akyuz L, et al. (2018) High prevalence of Streptococcus pyogenes Cas9-reactive T cells within the adult human population. Nature Medicine 25(2): 242 248.

106. Liang P, Xu Y, Zhang X, Ding C, (2015) CRISPR/Cas9-mediated gene editing in human tripronuclear zygotes. Protein Cell 6(5): 363-372. 